\title{
Editorial: Mollicutes: what are they?
}

An article in this issue (page 865) is concerned with the possibility that mollicutes are involved in the pathogenesis of so-called orbital pseudotumours. But what are mollicutes?

Delving into the literature reveals that they are a class of micro-organisms in the Prokaryote kingdom, that is, they are unicellular and, though they contain chromatin material, they do not have a distinct nucleus. There are two types or divisions of Prokaryote: Photobacteria, which are sensitive to light, and Scotobacteria, which are not. Of the Scotobacteria there are three classes: Bacteria, Rickettsia, and Mollicutes, the last being distinguished and defined by the absence of an outer cell wall. Hence the source of their name, from the Latin mollis, soft and cutis, skin. They are the smallest category of organism capable of growth in cell-free media.

Their existence has been known for some time although only in the last 10-15 years has their taxonomic position been clarified. Previously, mollicutes commonly went by the name of mycoplasmas, and it is still common practice for them to do so, but more recently it has become proper to reserve the term Mycoplasma for a defined genus within the Mollicute class. Many of the mycoplasmas and related genera are further distinguished by having a low proportion of guanosine plus cytosine in the base pairs of their cytoplasmic DNA and having a need of sterols, such as cholesterol, for growth.

In appearance those of relevance to the animal kingdom measure about $300 \mu \mathrm{m}$, are bounded by a single unit membrane but have no wall, are variably coccoid or filamentous in shape, and have a cytoplasm composed for the most part of ribosomes and diffuse or filamentous chromatin.

What of their relevance to human pathology in general and to ophthalmology in particular? About a dozen different Mollicutes, mostly Mycoplasmas and related genera, have been identified in human tissues, most occurring as part of the normal flora coating the several mucosal surfaces and, as such, of little or no clinical consequence. Nevertheless, occasionally they may be associated with opportunistic infections of the mouth and urogenital tract. Mycoplasma pneumoniae, on the other hand, is an undoubted if weak pathogen in that is is a frequent cause of atypical pneumonia in young people. Another Mollicute of tick origin is cataractogenic when injected into suckling mice, but reports of ocular and adnexal disease due to mycoplasmas and related organisms are sparse, being restricted to accounts of conjunctivitis in the newborn and, with iridocyclitis, in Reiter's syndrome.

It is of some interest, therefore, that structures comparable to Mollicutes are now described in the cytoplasm of leucocytes present in biopsies from patients with inflammatory orbital disease, given that the aetiology of most so-called pseudotumours is at present quite obscure. The authors prefer the term mollicute-like organism, since the putative pathogen resists attempts at cultivation and is identifiable on ultrastructural criteria alone. Whether the entities described are indeed viable organisms involved in chronic orbital inflammation and, if so, whether they are causal or merely coincidental remains to be seen.

A GARNER 